\title{
RESULTS FROM LUMINOSITY SCANS DURING THE RHIC 2000 RUN*
}

\author{
A. Drees, Brookhaven National Laboratory, Upton, NY 11973, USA \\ Zhangbu Xu, Yale University
}

\begin{abstract}
During the year 2000 run a total of eight beam scans (Vernier Scans) were performed at various interaction points (IP) at RHIC. During a Vernier Scan the experimental collision rates are recorded while the beams are stepwise scanned across each other. Vernier Scans yield transverse beam sizes as well as maximum luminosity and thus the absolute cross section, which with the limited data from the 2000 run we measured to be $\sigma=8.9 \pm 0.3$ barn at $\sqrt{s_{N N}}=130 \mathrm{GeV}$. Also, Vernier Scans permit performance studies of the beam orbit control and local coupling.
\end{abstract}

\section{INTRODUCTION}

The delivered total luminosity is an important parameter to determine the collider performance. In addition, the total cross section, which is derived from the luminosity measurement, is one of the necessary global event features used to normalize parameters of the initial state in heavy ion collisions such as the charged multiplicity. In this report, we describe the method of Vernier Scans to measure the absolute cross section of $\mathrm{Au}+\mathrm{Au}$ collisions at $\sqrt{s_{N N}}=130$ $\mathrm{GeV}$ at the Relativistic Heavy Ion Collider (RHIC). Because the experiments used ZDC (Zero Degree Calorimeter) [1] coincidences as a minimum bias trigger, the total absolute cross section presented here is the total cross section seen by the $\mathrm{ZDC}$ coincidences from $\mathrm{Au}+\mathrm{Au}$ interactions which include minimum bias nuclear interactions and mutual Coulomb Dissociation[2]. This cross section has been calculated in [2] resulting in $10.7 \pm 0.5 \mathrm{~b}$ at $\sqrt{s}=130$ $\mathrm{GeV}$ and deviates from the measured value. This discrepancy is not yet understood and will be investigated during the upcoming run 2001.

\section{THE ZERO DEGREE CALORIMETER}

The Zero Degree Calorimeter (ZDC) [1] is designed of alternating absorber and Cerenkov fiber layers. Crossing particles within a limited angular cone produce Cerenkov radiation which is channeled in the fiber ribbons to a photo multiplier tube. The ZDC units are located behind the DX magnets between the beam pipes at about $+/-18 \mathrm{~m}$ from each of the four equipped IPs subtending an angle of about $2.5 \mathrm{mrad}$ of the forward direction. Since charged particles are bent by the DX magnets they are sensitive to only uncharged particles from the interaction zone. The calorimeters are designed to measure neutrons emitted from nuclear fragments from $\mathrm{Au}+\mathrm{Au}$ collisions that missed the actual interaction zone. The inclusive correlated emission in each

* Work performed under the auspices of the US department of Energy.

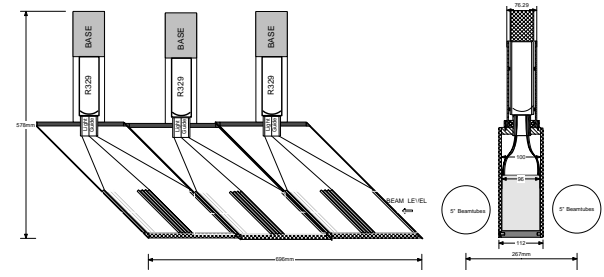

Figure 1: Front and side view of the 3 ZDC modules. 3 modules form one detector unit, of which 8 are installed in RHIC.

beam direction is used to suppress many kinds of backgrounds and corresponds to a large cross section, which has been calculated and is measured in this report.

\section{VERNIER SCANS}

The Vernier Scan was invented by S. van der Meer in 1968[3] who showed that it is possible to measure the effective height $h_{e}$ of the colliding ISR beams by observing the counting rate $R$ in a suitable monitor system while sweeping the two beams vertically through each other. A Gaussshaped curve results with its maximum at zero displacement. At RHIC the transverse displacement was achieved by applying orbit-bumps [5] around the IP. The typical step size was $200 \mu \mathrm{m}$ which could be confirmed by reconstructed vertices from the STAR TPC detector as shown in figure 2.



Figure 2: RHIC predicted beam set position and mean of STAR tracking primary vertex. The horizontal axis is time in units of minutes of the operation and vertical axis is mean value of twobeam overlapping region in units of $\mathrm{cm}$. The agreement between these two is considered to be very good.

The interaction rate $N_{Z D C}$ is defined as the number of beam particles $\left(N_{b l u}\right.$ and $\left.N_{y e l}\right)$ going through each other in some area $A$ with cross section $\sigma$ :

$$
N_{Z D C}=\frac{N_{b l u} N_{y e l}}{A} \sigma=\mathcal{L} \sigma
$$


For two beams with Gaussian distribution in both, horizontal and vertical directions, the luminosity is given by [4]:

$$
\mathcal{L}=\frac{k_{b} f_{\text {rev }} N_{1} N_{2}}{2 \pi \sqrt{\left(\sigma_{x 1}^{2}+\sigma_{x 2}^{2}\right)\left(\sigma_{y 1}^{2}+\sigma_{y 2}^{2}\right)}} .
$$

with $\mathrm{i}=1,2$ for blue and yellow beams respectively and $N_{i}$ the number of particles per bunch assuming all bunches in one beam are the same. Bunch-to-bunch variations will be discussed below in section 5 . In the case of non-centered beams, consider the case of one beam displaced by $\mathrm{d}$, the luminosity $\mathcal{L}(d)$ as a function of $\mathrm{d}$ is:

$$
\mathcal{L}(d)=\frac{k_{b} f_{r e v} N_{1} N_{2} \exp \left[-d^{2} / 2\left(\sigma_{x 1}^{2}+\sigma_{x 2}^{2}\right)\right]}{2 \pi \sqrt{\left(\sigma_{x 1}^{2}+\sigma_{x 2}^{2}\right)\left(\sigma_{y 1}^{2}+\sigma_{y 2}^{2}\right)}}
$$

The terms $\sqrt{\sigma_{i 1}^{2}+\sigma_{i 2}^{2}}$, where $i=x, y$, in Eq.3 and Eq.2 correspond to the beam profile derived from the width of the distribution measured by the Vernier Scan. This results in:

$$
\sigma_{V x}=\sqrt{\sigma_{x 1}^{2}+\sigma_{x 2}^{2}} .
$$

Vernier Scans measure the effective beam area over the whole longitudinal interaction range, i.e. approximately + $5 \mathrm{~m}$, instead of measuring the beam transverse size at the center of the IP with the corresponding values of the betafunction, $\beta^{*}=8 \mathrm{~m}^{1}$ and $\beta^{*}=3 \mathrm{~m}^{2}$. However, the collision rates we get from the scans correspond to the entire effective beam area as well.

\section{DATA ANALYSIS}

A total of eight Vernier Scans was done at PHENIX (IP 8), PHOBOS (IP 10) and STAR (IP 6) during the year 2000 run. Table 1 summarizes all scans. Figure 3 shows the horizontal and vertical distributions from the scan at PHENIX. The archived distributions were fitted to a Gauss function

\begin{tabular}{|c|l|c|c|c|c|}
\hline$\#$ & $\mathrm{IP}$ & $\beta^{*}[\mathrm{~m}]$ & start time & end time & $t_{s t}[\mathrm{~h}]$ \\
\hline 1 & 8 & 3 & $08 / 22,07: 55$ & $08 / 22,08: 35$ & 1.9 \\
\hline 2 & 10 & 8 & $08 / 23,21: 55$ & $08 / 23,22: 28$ & 1.4 \\
3 & 10 & 8 & $08 / 23,23: 52$ & $08 / 24,00: 17$ & 3.3 \\
\hline 4 & 6 & 8 & $08 / 25,18: 00$ & $08 / 25,19: 42$ & 5.1 \\
\hline 5 & 10 & 8 & $08 / 29,17: 03$ & $08 / 29,17: 35$ & 2.8 \\
6 & 10 & 8 & $08 / 29,19: 49$ & $08 / 29,20: 17$ & 5.5 \\
\hline 7 & 6 & 8 & $08 / 30,01: 20$ & $08 / 30,02: 30$ & 1.0 \\
8 & 6 & 8 & $08 / 30,05: 00$ & $08 / 30,06: 25$ & 4.8 \\
\hline
\end{tabular}

Table 1: List of vernier scans performed during the year 2000 RHIC run. Scans performed during the same store are grouped together. $t_{s t}$ is the (center-)time of the scan with respect to the start of the fill, i.e. the time when the flat-top energy is reached.

yielding the following results:

- horizontal and vertical beam sizes $\sigma_{V x}, \sigma_{V y}$

- location of fully overlapping beams (center location) in the two planes, $x_{\max }$ and $y_{\max }$

\footnotetext{
${ }^{1}$ STAR and PHOBOS

${ }^{2}$ PHENIX and BRAHMS
}
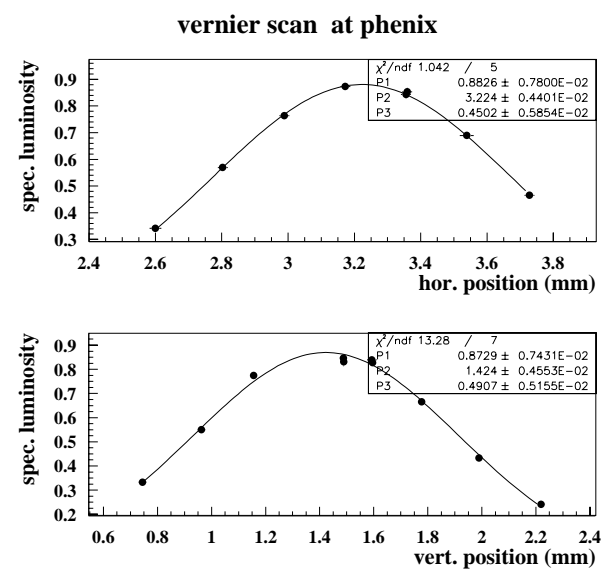

Figure 3: A Vernier Scan at the PHENIX interaction point with $\beta^{*}=3 \mathrm{~m}$. The Gauss-fit is superimposed, errors are statistical.

- maximum collision rates from the 2 fits:

$R_{\max }^{x}=N_{Z D C}^{x} / N_{b l u} N_{y e l}$

$R_{\max }^{y}=N_{Z D C}^{y} / N_{b l u} N_{y e l}$

Table 2 summarizes the uncorrected fit results for all scans.

\begin{tabular}{|c|c|c|c|c|c|c|c|}
\hline id & $\sigma_{V x}$ & $\delta_{V x}$ & $\sigma_{V y}$ & $\delta \sigma_{V y}$ & $R_{\max }^{x}$ & $R_{\max }^{y}$ & $\delta R_{\max }$ \\
\hline 1 & 449 & 6 & 487 & 5 & 0.846 & 0.830 & 0.008 \\
2 & 715 & 13 & 685 & 13 & 0.427 & 0.407 & 0.004 \\
3 & 781 & 30 & 722 & 20 & 0.328 & 0.323 & 0.004 \\
4 & 932 & 24 & 769 & 30 & 0.211 & 0.292 & 0.007 \\
5 & 885 & 28 & 707 & 16 & 0.296 & 0.316 & 0.003 \\
6 & 1180 & 104 & 763 & 40 & 0.283 & 0.299 & 0.005 \\
7 & 658 & 5 & 607 & 6 & 0.415 & 0.411 & 0.007 \\
8 & 916 & 11 & 734 & 13 & 0.264 & 0.268 & 0.005 \\
\hline
\end{tabular}

Table 2: Uncorrected fit results from the eight Vernier Scans, given errors are statistical. The widths are given in units of $\mu \mathrm{m}$, the normalized rates in $10^{-18} \mathrm{~Hz}$.

\section{CROSS SECTIONS}

Several mechanisms can adulterate the cross section results:

- The WCM calibration has to be understood.

- The filling pattern and the actual colliding bunches change the effective beam current causing $R_{\max }$ to increase.

- Beam blow-up [6] is a continuous process causing the normalized collision rate to decrease as a function of store time resulting in $R_{\max }^{y}$ being systematically lower than $R_{\text {max }}^{y}$.

- In addition to the continuous blow-up there could be other effects increasing the beam size such as the scan itself when beams are typically steered off-center more than usual. This again would result in $R_{\max }^{y}$ being smaller than $R_{\max }^{x}$. 
- Before a scan is done the center location is not known in either plane, hence the vertical position is likely to be off-center, reducing $R_{\max }^{x}$.

- Coupling changes the position in the non-swept plane while a scan is in progress in the other. This would reduce the maximum achievable counting rate even for perfectly head-on beams.

- After a scan is performed in one plane beams were steered with limited accuracy to a head-on position.

The WCM [7] readings are adjusted to the high precision DCCT [8] current measurement using data from the beginning of the store only since the WCM is not sensitive to debunched beam. The fill pattern was recorded by the WCM during every store, one example shown in figure 4 , and was used to correct $N_{1} N_{2}$. The beam position was recorded in
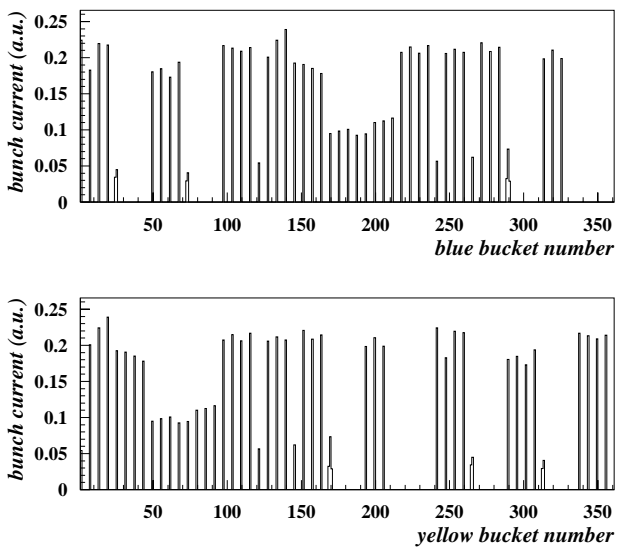

Figure 4: Fill pattern as measured with the WCM in the blue and yellow ring. Reference point is the PHENIX IP. The bucket number in the yellow ring is shifted by 20 such that the yellow bucket \# 21 corresponds to bucket \# 1 according to actually colliding pairs.

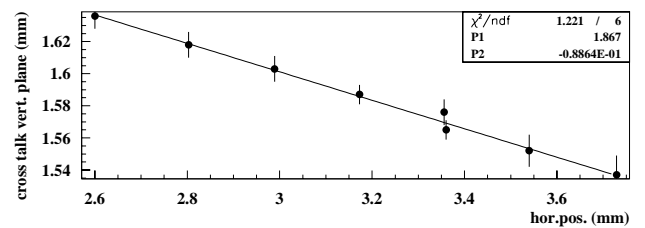

Figure 5: Measured movement in the vertical plane at PHENIX while scanning the horizontal plane.

both planes at any time during the scans. From this data, a correction of $R_{\max }$ for mis-steering in the non-swept plane, such as a cross talk between the two (figure 5), could be derived. The absolute cross sections in table 3 are calculated according to: $\sigma_{A u+A u}=R_{\max } \frac{2 \pi \sigma_{V x} \sigma_{V y} k_{b}}{f_{\text {rev }}}$. Here, $R_{\max }$ and $\sigma_{V x}, \sigma_{V y}$ are the Gauss fit parameters.

\section{SUMMARY}

Vernier Scans are a useful tool to determine the total cross section as well as for emittance measurements, luminosity measurements and IP optimization. The total measured cross section from the ZDCs as presented in this report is $8.9 \pm 0.3 \mathrm{~b}$ (preliminary). The discrepancy from the

\begin{tabular}{|l|c|c|c|c|c|}
\hline id & $\begin{array}{c}R_{\max } \\
10^{-18} \mathrm{~Hz}\end{array}$ & $\begin{array}{c}\sigma_{V x} \\
\mu m\end{array}$ & $\begin{array}{c}\sigma_{V y} \\
\mu m\end{array}$ & $\begin{array}{c}\text { CS 1 } \\
\text { barn }\end{array}$ & $\begin{array}{c}\text { CS 2 } \\
\text { barn }\end{array}$ \\
\hline 1 & 0.883 & 450 & 491 & 8.7 & 9.9 \\
\hline 2 & 0.433 & 715 & 685 & 9.4 & 9.4 \\
\hline 3 & 0.334 & 782 & 722 & 8.4 & 8.4 \\
$4(*)$ & 0.292 & 932 & 768 & 9.4 & 9.5 \\
\hline 5 & 0.318 & 885 & 707 & 8.8 & 8.8 \\
6 & 0.300 & 1179 & 764 & 12.0 & 12.0 \\
\hline 7 & 0.460 & 657 & 607 & 8.2 & 8.0 \\
8 & 0.289 & 917 & 734 & 8.6 & 8.5 \\
\hline
\end{tabular}

Table 3: List of Vernier Scans and resulting total cross sections (CS) after correcting for being off-center in the plane which is not scanned (CS 1) and after correcting for the fill pattern (CS 2). (*) CS 1 is uncorrected for this scan since there was no data available.

predicted value $10.7 \pm 0.5 \mathrm{~b}$ is not yet understood and the analysis is still in progress. For better understanding and in order to reduce the statistical uncertainty more Vernier Scans with better data acquisition tools will be performed in the upcoming year 2001 run.

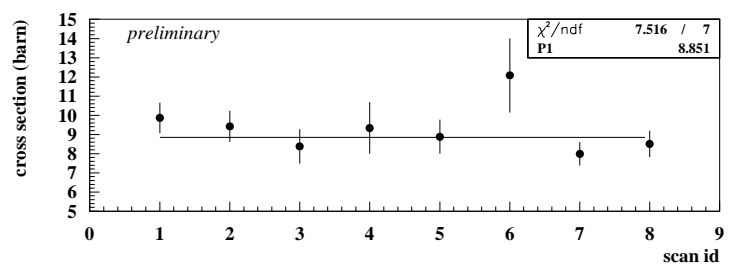

Figure 6: Corrected cross sections from the eight vernier scans. Errors shown include statistical and systemactic error sources.

\section{ACKNOWLEDGEMENTS}

The authors are thankful to the the ZDC collaboration for their efforts in designing, building, installing and maintaining the calorimeters. We also thank operations, instrumentation, radio frequency, control and the accelerator physics teams that supported the data acquisition, storage, processing and analysis in many ways. We also thank J. Sandweiss for many useful discussions.

\section{REFERENCES}

[1] C. Adler, et al., nucl-ex/0008005.

[2] A. Baltz, et al., Nucl. Instr. and Method, A417 (1998) 1

[3] S. van der Meer, ISR-PO/68-31, KEK68-64.

[4] P. Castro-Garcia, CERN SL/96-70, KEK97-01-158.

[5] S. Peggs et al., "Interaction Region Closed Orbits", internal note RHIC/AP/135, December 1997.

[6] W. Fischer, A. Drees et al., "Beam Lifetime and Emittance Growth Measurements of Gold Beams in RHIC at Storage", this proceedings

[7] P. Cameron et al., "The RHIC Wall Current Monitor System", Proceedings of the 1999 PAC, p. 2146 (1999).

[8] Bergoz Instrumentation, "http://bergoz.com”, Beam Charge Monitors 